Scientific Journal of

Basic and Applied Sciences

Available online at Science-Tanta

Research Article

BIOCHEMISTRY

\title{
Effect of combined Hesperidin, Piperine, Bee venom and Tamoxifen on oxidative stress and hepato-nephrotoxicity amelioration against breast cancer in rats
}

\author{
Abeer A. Khamis ${ }^{1 *}$, Ehab M. Ali ${ }^{1,2}$, Mohamed A. Abd El-Moneim³ ${ }^{3}$ Elsayed I. Salim ${ }^{4}$ \\ 1. Biochemistry Division, Chemistry Department, Faculty of Science, Tanta University, Tanta 31527, Egypt \\ 2. Biochemistry Division, Chemistry Department, Faculty of Science, King Abdul Aziz. University KAU, Jeddah, \\ Saudi Arabia \\ 3. Biochemistry Department, Faculty of Dentistry, Sinai University, Al-Arish, North Sinai, Egypt \\ 4. Zoology Department, Faculty of Science, Tanta University, Tanta, Egypt.
}

* Correspondence: Abeer A. Khamis, Biochemistry Division, Chemistry Department, Faculty of Science, Tanta University, Tanta 31527,Egypt; email: abeer.khamis@science.tanta.edu.eg

\section{KEY WORDS}

Breast cancer;

Tamoxifen;

Hesperidin;

Piperine;

Bee venom;

Hepato-nephrotoxicity

\begin{abstract}
Breast cancer is the most commonly diagnosed cancer and the major cause of cancer-related death among females. Studies on natural compounds used alone or in combination with chemotherapeutic agents proved their efficacy to treat and prevent cancer. Hesperidin, Piperine, and Bee venom displayed anti-oxidant and anti- cancer activity against various types of cancers and cell lines and can be used in combination with Tamoxifen with the aim to decrease cytotoxicity profile. In-vivo study of Piperine (Pip), Bee venom (BV) and hesperidin (Hes) as a single treatment or in combination with/without tamoxifen indicated low hepato-nephrotoxicity and hepatonephroprotective against tamoxifen-treated and untreated xenograft MCF-7 induced breast cancer. Serum ALT, AST, ALP activities and creatinine, and urea level were elevated. MDA level was increased with a marked reduction in SOD activity in the untreated xenograft MCF-7 induced breast cancer. Treatments for these natural products improve these parameters. It could be concluded that alone or in combination with/ without Tamoxifen clarified a modulatory role against breast cancer.
\end{abstract}

\section{Introduction}

Breast cancer prevalence is substantially serious. The incidence and mortality of breast cancer are 43.3 and 12.9 per million in women respectively, which is in the top of all female related cancers [1]. Breast cancer is controlled by surgery, chemotherapy, radiotherapy and frequently supported by hormone therapies [2].
Even though treatment strategies of breast cancer have been developed, current treatments of breast cancer are still not enough to fundamentally treat this disease due to; side effects, drug resistance and non-specificity [3]. This situation pushes researcher to keep moving to discover new promising anticancer drugs. 
Numerous modalities for treatment of cancer are available for cancer such as radiation therapy, chemotherapy, immunotherapy surgery, and hormonal therapy. Tamoxifen (TAM), a nonsteroidal anti-estrogen drug, is widely used for the treatment of patients with all stages of estrogen-dependent breast cancer, and the long-term single use of tamoxifen has been applied to treat patients during the premenstrual period [4]. For women with ERpositive breast cancer, treatment for 5 years with adjuvant tamoxifen substantially reduces the rate of recurrence [5]. Recent trials have shown that continuing tamoxifen for 10 years rather than stopping at 5 years produces a further reduction in recurrence and mortality [6]. However, many ER-positive patients are low reactive or resistant to tamoxifen and such long treatment with tamoxifen causes serious side-effects such as increases in endometrial hyperplasia and carcinomas, an excess of venothrombotic episodes, and the development of de novo or acquired tamoxifen resistance [5]. Thus, there is a need for more effective therapy with fewer side-effects. Several natural products including hesperidin, piperine, and bee venom have served as anticancer where they used alone or in combination with anticancer drugs hesperidin (Hes) is one of the most abundant natural flavonoids and is present in many fruits and vegetables.

Some authors reported that Hes prevents oxidant injury and cell death by several mechanisms, such as scavenging oxygen radicals and protecting against lipid peroxidation and chelating metal ions [10]. Hesperidin has a non-toxic effect on normal cells, but it can suppress cell proliferation in several cancer types and so it acts as an anticarcinogenic agent in some tumors. In addition, hesperidin as a radio-protective and the chemo-protective therapeutic agent is expected to prevent invasion or metastasis of human cancers [11]. Anti-cancer effects of hesperidin were studied in tumor-implanted animal models or culture cell lines of several cancer types, including colon cancer, bladder cancer, hepatocarcinoma cancer, and breast cancer [11].

Piperine (Pip) is the main phenolic ingredient of black pepper [17], exhibits various activities including anti-tumor, anti-inflammatory, immunomodulatory, anti-metastatic and hepatoprotective activities. It possesses antioxidative, and chemo-protective property [18, 19]. It also exerts anti-cancer effects and antiinvasion activity with less toxicity [20]. Piperine inhibits lung metastasis induced by B16F-10 melanoma cells and also inhibits fibrosarcoma cells invasion [21].

Bee venom (BV) treatment is the therapeutic application of honeybee venom to treat the various diseases. BV has been used in Oriental medicine to treat a variety of conditions, such as arthritis, rheumatism, back pain, cancerous tumors, and skin diseases. BV contains at least 18 active components, including melittin, apamin, adolapin, the mast-cell-degranulating (MCD) peptide, enzymes (i.e., phospholipase [PL] A2), biologically active amines (i.e., histamine and epinephrine), and nonpeptide components which have a variety of pharmaceutical properties [22]. Many studies investigated its anti-inflammatory, antirheumatoid arthritis and immune modulatory effects [23, 24]. Bee venom has also been reported to enhance the apoptotic signaling pathway in experimental osteosarcoma, breast cancer, and lung cancer cell lines [24, 25]. Induction of apoptosis by melittin or Bee venom has been reported in human lung cancer [23], rheumatoid arthritis [24], hepatocellular carcinoma [26], human leukemic cells [25], human breast cancer [27], prostate [28] and ovarian cancer cells [29]. Modulation of Tamoxifen sensitivity that results in lessening of its side-effects, resistance and maximizes its therapeutic efficiency is a desirable goal. The combination therapy with potent chemotherapeutic agents from natural compounds may achieve synergistic therapeutic effect, dose and toxicity reduction, and to minimize or delay the induction of drug resistance.

However, to date, there has been no study on oxidative stress and hepato-nephrotoxicity modulation effects of tamoxifen in combination with the three natural products Hes, Pip, and BV. Therefore, this study has focused on evaluating the oxidative stress and hepato-nephrotoxicity amelioration of tamoxifen and the three natural products against breast cancer MCF7 cells. 


\section{Materials and Methods}

2.1. Animals

One hundred and thirty-two healthy female albino rats aged 4 weeks and weighted approx. 150- $170 \mathrm{gm}$ were housed in a temperaturecontrolled and light-controlled room $(12 \mathrm{~h}$ light/dark cycle) with free access to food and water. All experimental procedures followed the guidelines of the Institutional Animal Care and Use Committee of the Faculty of Science, Tanta University. Rats were acclimatized to laboratory conditions for two weeks prior to experiments [30].

\subsection{Induction of breast cancer model by xenografting of MCF7 cells}

The rats were immunosuppressed by intraperitoneal injection of cyclophosphamide (CTX) at a dose of $40 \mathrm{mg} / \mathrm{kg}$ for 4 successive days. To establish the breast cancer model, a human breast cancer cell line cells (MCF7) were counted and injected intradermally around the teat of the mammary gland at a dose of $1 \times 10^{7}$ cells in a volume of $1 \mathrm{ml}$ [31]. To initiate tumor formation, rats were injected intra muscularly by estrogen (Folon, 0.01 $\mathrm{ml} / \mathrm{kg}$ body weight) one day before MCF7 injection, and continued daily till tumor formation (approximately 4 weeks).

\subsection{Groups}

The 132 rats were equally divided (12 per group) into the following groups: Group 1: Healthy control group was orally administered with $1 \mathrm{ml} 0.9 \%$ saline and intraperitoneally injected with $0.5 \mathrm{ml}$ saline. Group 2: Breast cancer control group. Group 3: Breast cancer rats orally (by gastric tube) administrated tamoxifen (Nolvadex®, Astra Zenca Cambridge, UK) with a dose equal to $5 \mathrm{mg} / \mathrm{kg}$ once daily [32]. Group 4: Breast cancer rats treated before and with induction of breast cancer with hesperidin (Acros Organics, USA; Cat no. 123460050), piperine (Acros Organics, USA; Cat no. 381450050) and bee venom (Apis Injeel TM, Heel GMBH, Germany) and divided into 3 sub-groups: Sub-group 4A: Breast cancer rats orally (by gastric tube) administrated hesperidin with a dose 200mg/kg once daily [33]. Sub-group 4B: Breast cancer rats orally (by gastric tube) administrated piperine with a dose equal to $50 \mathrm{mg} / \mathrm{kg}$ once daily [34]. Sub group 4C: Breast cancer rats intraperitoneally injected by bee venom (BV) with a dose equal to $0.5 \mathrm{mg} / \mathrm{k} . \mathrm{g}$ once daily $[35,36]$. Group 5 and group 6: Breast cancer rats treated before and with induction of breast cancer with combination of Hes, Pip and BV alone or in combination with Tam with the best selected ratio in vitro study as the following: Group 5: divided into 2 sub-groups Sub-group 5A: Breast cancer rats treated by Tam and Pip with a ratio of 1:4, respectively. Sub-group 5B: Breast cancer rats treated by Hes, Pip, and BV with a ratio of 1:2:1, respectively. Group 6: divided into 2 sub-groups: Sub group 6A: Breast cancer rats treated by Tam and Pip with a ratio of 1:4, respectively. Sub-group 6B: Breast cancer rats treated by Tam, Pip, and BV with a ratio of 1:2:1, respectively. Sub-group 6C: Breast cancer rats treated by Tam, Hes, Pip, and BV with a ratio of $1: 1: 4: 1$, respectively.

\subsection{Sample collection}

Blood samples were collected from the medial canthus venous plexus into dry clean centrifuge tubes. The tubes were kept at room temperature for 15 minutes for blood clotting and centrifuged at $3000 \mathrm{rpm}$ for 10 minutes. The sera were separated and were kept at $20^{\circ} \mathrm{C}$ until analyzed for biochemical estimations. Mammary glands were immediately excised under sterilized conditions and snap-frozen in liquid nitrogen and stored at $-80{ }^{\circ} \mathrm{C}$ for further use.

\subsection{Biochemical analysis}

In the normal and tumorous mammary gland homogenate, Serum ALT and AST activities were determined by a colorimetric method which described by [39]. Serum ALP activity was measured according to the method of [40]. Serum creatinine level was determined by a colorimetric method which described by [41] was used. Serum urea level was determined by [42]. SOD activity was determined by the colorimetric method described by [37] and determination of MDA level was carried out according to the method of [38] was used.

\subsection{Statistical analysis}

Statistical analysis was performed using oneway analysis of variance (ANOVA) with Tukey-Kramer multiple comparisons test as a 
post ANOVA test. Significant differences among means were estimated at $\mathrm{p}<0.05$. The results were expressed as mean \pm SEM (standard error of the mean). Values were analyzed using Graph Pad Prism 5 (Graph Pad Software, Inc., LaJolla, CA, USA).

\section{Results}

3.1. Effect of treatments with tamoxifen, hesperidin, piperine, and bee venom on liver function (ALT, AST, ALP)

The serum activities of liver enzymes (AST, ALT and ALP were found to be significantly higher in xerographic MCF-7 injected rats when compared to normal control animals (Table 1, Fig. 1). These elevated enzymes were significantly reduced following treatment by tamoxifen, hesperidin, piperine, and bee venom alone or in combination with the best improvement in combined groups especially those including the four compounds.

3.2. Effect of treatments with tamoxifen, hesperidin, piperine, and bee venom on kidney function (urea and creatinine)

Urea and creatinine levels in serum of xerographic MCF-7 injected rat's groups showed a highly significant increase at when compared with the normal group. Those findings recorded in (Table 2, Fig. 2). Treated groups tamoxifen, hesperidin, piperine, and bee venom alone or in combination showed significant decreases at in serum creatinine and urea levels comparing to control animals with the best improvement in combined groups especially those including the four compounds.

Table 1: Effect of Tam, Hes, Pip, and BV on serum activities of liver enzymes.

\begin{tabular}{cccc}
\hline Groups & ALT(U/L) & AST(U/L) & ALP(U/L) \\
\hline G1: healthy control & $38.33^{\mathbf{g}} \pm 0.88$ & $36^{\mathbf{f}} \pm 1$ & $114.3^{\mathbf{f}} \pm 2.6$ \\
G2: breast cancer control & $106^{\mathbf{a}} \pm 1.53$ & $88.67^{\mathbf{a}} \pm 1.5$ & $223.3^{\mathbf{a}} \pm 2$ \\
G3: Tam & $101.83^{\mathbf{a}} \pm 2$ & $82^{\mathbf{a}} \pm 1.42$ & $216.7^{\mathbf{a}} \pm 3$ \\
G4A: Hes & $81.50^{\mathbf{b}} \pm 0.67$ & $59^{\mathbf{b}, \mathbf{c}} \pm 2.6$ & $183.3^{\mathbf{b}} \pm 2.3$ \\
G4B: Pip & $74.67^{\mathbf{c}} \pm 1.45$ & $53.67^{\mathbf{c}, \mathbf{d}} \pm 2.2$ & $177.7^{\mathbf{b}} \pm 2$ \\
G4C: BV & $81.73^{\mathbf{b}} \pm 0.67$ & $62^{\mathbf{b}} \pm 2.1$ & $183.3^{\mathbf{b}} \pm 2.3$ \\
G5A: Hes+Pip & $67.17^{\mathbf{d}} \pm 1.01$ & $48^{\mathbf{d}} \pm 2.4$ & $156.3^{\mathbf{c}} \pm 1.9$ \\
G5B: Hes+Pip+BV & $55.67^{\mathbf{e}} \pm 1.31$ & $45.67^{\mathbf{d}, \mathbf{e}} \pm 1.2$ & $142.3^{\mathbf{d}} \pm 1.5$ \\
G6A: Tam -pip & $64.67^{\mathbf{d}} \pm 1.45$ & $49.6^{\mathbf{d}} \pm 0.9$ & $156.7^{\mathbf{c}} \pm 1.9$ \\
G6B: Tam+Pip-BV & $53.13^{\mathbf{e}} \pm 1.04$ & $46.57^{\mathbf{d}, \mathbf{e}} \pm 1.5$ & $140.7^{\mathbf{d}} \pm 1.2$ \\
G6C: Tam+Hes+Pip+BV & $46.23^{\mathbf{f}} \pm 1.18$ & $39^{\mathbf{e}, \mathbf{f}} \pm 1.2$ & $128.7^{\mathbf{e}} \pm 0.9$ \\
\hline
\end{tabular}

N.B. Values in the same column with different superscript letters are significantly different $p$ values. Data are presented as mean \pm S.E.M $(n=12)$.

Table 2: Effect of tamoxifen, hesperidin, piperine, and bee venom on serum kidney parameters.

\begin{tabular}{ccc}
\hline Group & Urea $(\mathbf{m g} / \mathbf{d l})$ & Creatinine $(\mathbf{m g} / \mathbf{d l})$ \\
\hline G1: healthy control & $20.27^{\mathbf{e}} \pm 0.39$ & $0.87^{\mathbf{g}} \pm 0.01$ \\
G2: breast cancer control & $49.68^{\mathbf{a}} \pm 0.93$ & $2.73^{\mathbf{a}} \pm 0.03$ \\
G3: Tam & $47.14^{\mathbf{a}} \pm 1.01$ & $2.36^{\mathbf{b}} \pm 0.09$ \\
G4A: Hes & $32.34^{\mathbf{b}} \pm 0.48$ & $1.55^{\mathbf{c}, \mathbf{d}} \pm 0.03$ \\
G4B: Pip & $31.83^{\mathbf{b}} \pm 0.44$ & $1.3^{\mathbf{e}} \pm 0.06$ \\
G4C: BV & $32.23^{\mathbf{b}} \pm 0.54$ & $1.62^{\mathbf{c}} \pm 0.04$ \\
G5A: Hes+Pip & $30.97^{\mathbf{b}} \pm 0.75$ & $1.23^{\mathbf{e}} \pm 0.02$ \\
G5B: Hes+Pip+BV & $26.03^{\mathbf{d}} \pm 0.38$ & $1.16^{\mathbf{e}, \mathbf{f}} \pm 0.01$ \\
G6A: Tam -pip & $30.07^{\mathbf{b , c}} \pm 0.58$ & $1.36^{\mathbf{d , e}} \pm 0.02$ \\
G6B: Tam+Pip-BV & $27.47^{\mathbf{c , d}} \pm 0.27$ & $1.26^{\mathbf{e}} \pm 0.02$ \\
G6C: Tam+Hes+Pip+BV & $23^{\mathbf{e}} \pm 0.53$ & $11^{\mathbf{f , g}} \pm 0.06$ \\
\hline
\end{tabular}

Values in the same column with different superscript letters are significantly different $p$ values. Data are presented as mean \pm S.E.M $(n=12)$. 

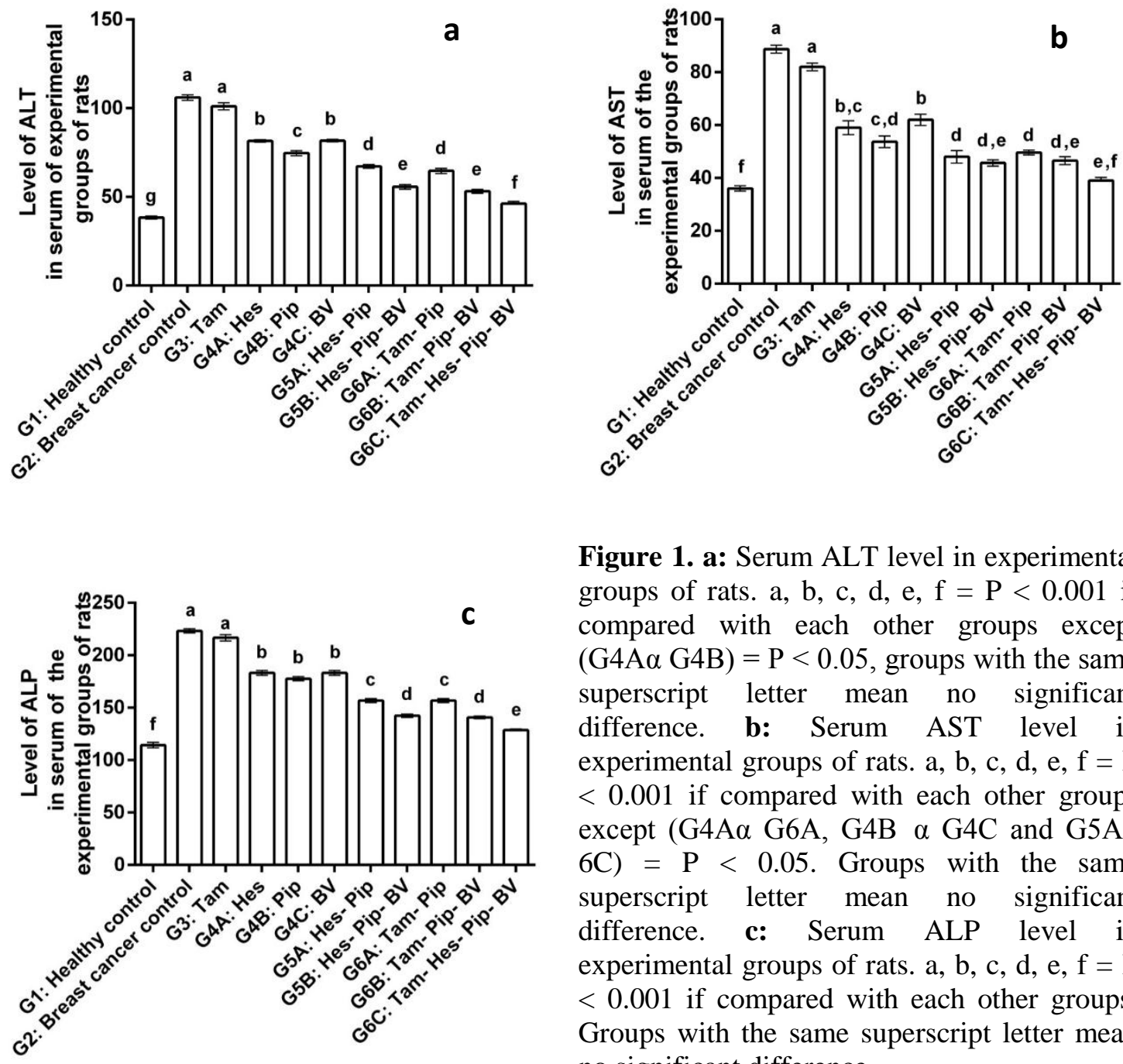

Figure 1. a: Serum ALT level in experimental groups of rats. a, b, c, d, e, f $=\mathrm{P}<0.001$ if compared with each other groups except $(\mathrm{G} 4 \mathrm{~A} \alpha \mathrm{G} 4 \mathrm{~B})=\mathrm{P}<0.05$, groups with the same superscript letter mean no significant difference. b: Serum AST level in experimental groups of rats. a, b, c, d, e, $\mathrm{f}=\mathrm{P}$ $<0.001$ if compared with each other groups except (G4A $\alpha$ G6A, G4B $\alpha$ G4C and G5A $\alpha$ $6 C)=P<0.05$. Groups with the same superscript letter mean no significant difference. c: Serum ALP level in experimental groups of rats. $a, b, c, d, e, f=P$ $<0.001$ if compared with each other groups. Groups with the same superscript letter mean no significant difference.
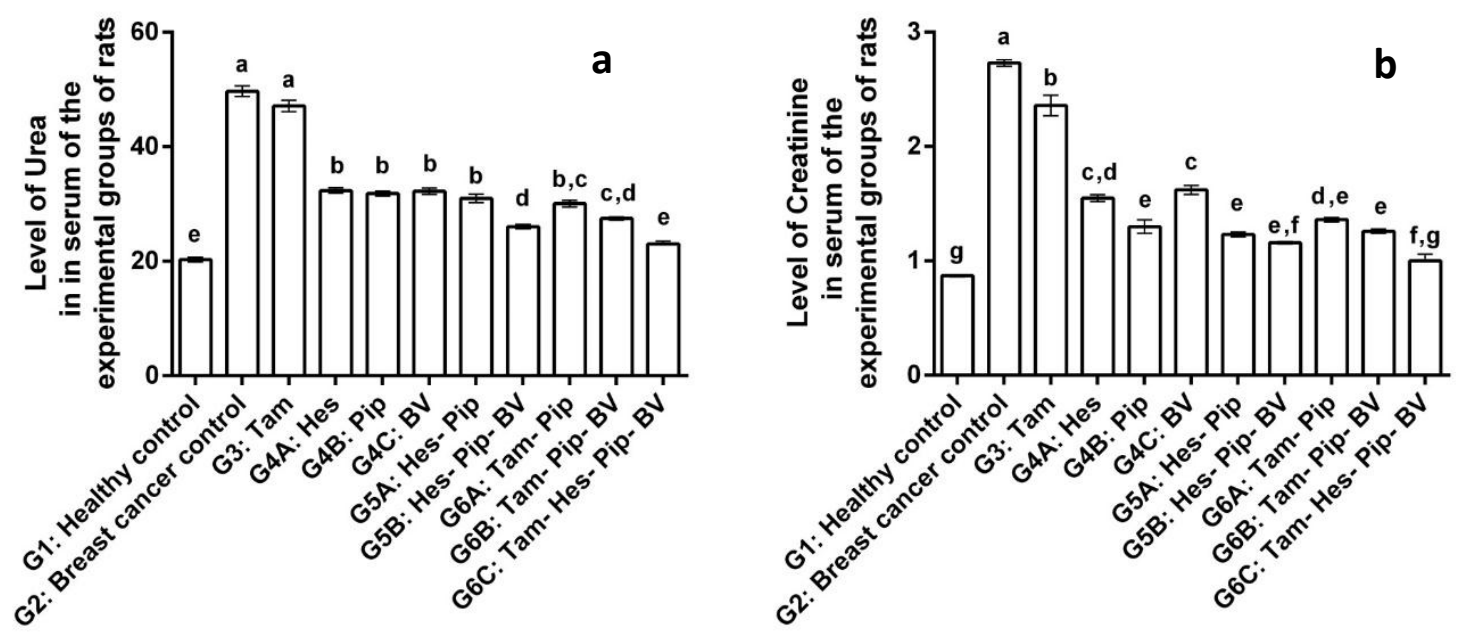

Figure 2. a: Serum Urea level in experimental groups of rats a, b, c, d, e $=P<0.001$ if compared with each other groups except $(\mathrm{G} 5 \mathrm{~B} \alpha \mathrm{G} 6 \mathrm{C})=\mathrm{P}<0.05$. Groups with the same superscript letter mean no significant difference. b: Serum creatinine level in experimental groups of rats $a, b, c, d, e, f=P<0.001$ if compared with each other groups except (G5A $\alpha$ G6C) $=\mathrm{P}<0.05$. Groups with the same superscript letter mean no significant difference. 


\subsection{Effect of treatments with tamoxifen,} hesperidin, piperine, and bee venom on MDA and SOD levels in mammary glands

The mammary glands of xerographic MCF-7injected rats had a significantly higher MDA level and a significant lower SOD activity as compared to normal control animals (Table 3, Fig. 3). Administration of tamoxifen, hesperidin, piperine, and bee venom alone or in combination improved the disrupted level of MDA and SOD with the best improvement in combined groups especially those including the four compounds.

Table 3: Effect of Tam, Hes, Pip, and BV on the MDA and SOD levels in normal and tumorous mammary glands.

\begin{tabular}{ccc}
\hline Group & MDA (nM/g protein) & SOD (IU/g protein) \\
\hline G1: healthy control & $30.67^{\mathbf{f}} \pm 0.88$ & $0.95^{\mathbf{a}} \pm 0.03$ \\
G2: breast cancer control & $110.8^{\mathbf{a}} \pm 5.42$ & $0.04^{\mathbf{g}} \pm 00$ \\
G3: Tam & $66.5^{\mathbf{b}, \mathbf{c}} \pm 2.90$ & $0.3^{\mathbf{e}} \pm 0.01$ \\
G4A: Hes & $69.7^{\mathbf{b}} \pm 2.93$ & $0.22^{\mathbf{f}} \pm 0.02$ \\
G4B: Pip & $68.63^{\mathbf{b}} \pm 2$ & $032^{\mathbf{e}} \pm 0.01$ \\
G4C: BV & $70.33^{\mathbf{b}} \pm 2.03$ & $0.2^{\mathbf{f}} \pm 0.01$ \\
G5A: Hes+Pip & $58.03^{\mathbf{c}, \mathbf{d}} \pm 1.25$ & $0.48^{\mathbf{d}} \pm 0.01$ \\
G5B: Hes+Pip+BV & $46.5^{\mathbf{e}} \pm 0.89$ & $0.60^{\mathbf{c}} \pm 0.01$ \\
G6A: Tam -pip & $59.05^{\mathbf{b}, \mathbf{d}} \pm 1.75$ & $0.45^{\mathbf{d}} \pm 0.01$ \\
G6B: Tam+Pip-BV & $51.47^{\mathbf{d , e},} \pm 0.87$ & $0.62^{\mathbf{c}} \pm 0.03$ \\
G6C: Tam+Hes+Pip+BV & $40.6^{\mathbf{e}, \mathbf{f}} \pm 0.92$ & $0.78^{\mathbf{b}} \pm 0.01$ \\
\hline
\end{tabular}

Values in the same column with different superscript letters are significantly different $p$ values. Data are presented as mean \pm S.E.M $(n=12)$.
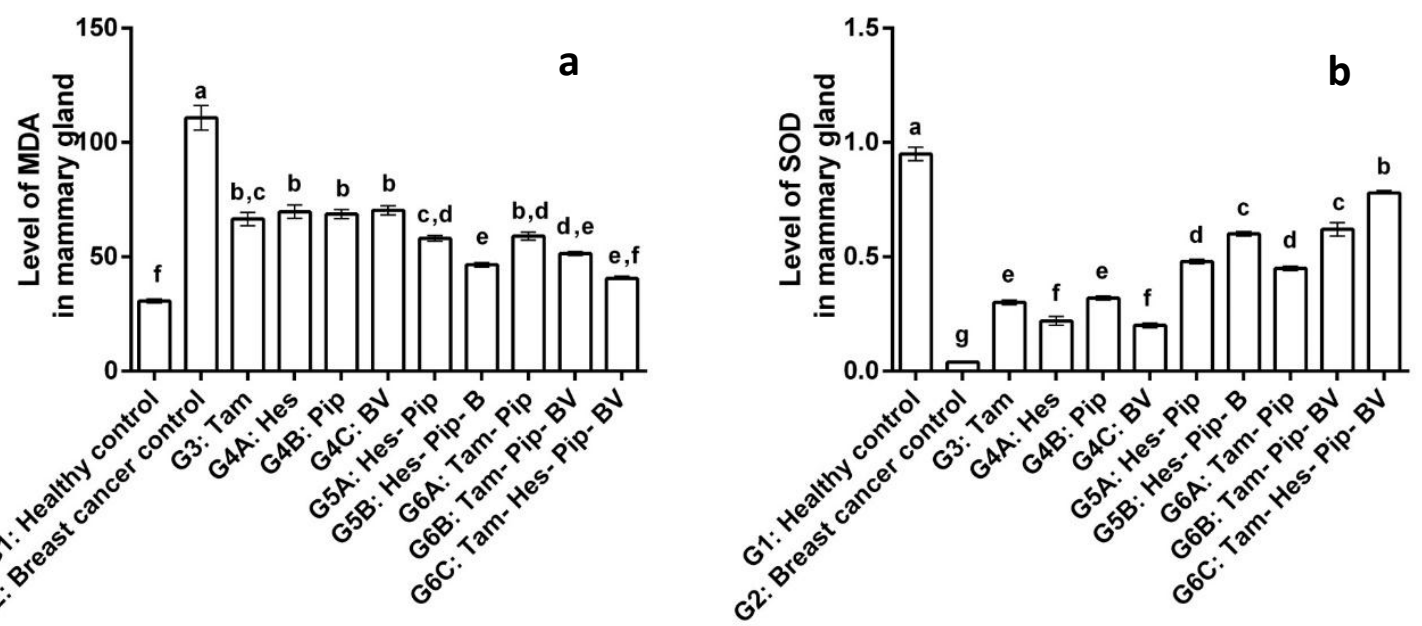

Figure 3. a: MDA level in tumorous mammary gland of rats a, b, c, d, e $=P<0.001$ if compared with each other groups except $(\mathrm{G} 5 \mathrm{~B} \alpha \mathrm{G} 6 \mathrm{C})=\mathrm{P}<0.05$. Groups with the same superscript letter mean no significant difference except (G4A $\alpha$ G5A, G4C $\alpha$ G5A, G5A $\alpha$ G5B and G5B $\alpha$ G6A) = $\mathrm{P}<0.05$. b: SOD level in tumorous mammary gland of rats $\mathrm{a}, \mathrm{b}, \mathrm{c}, \mathrm{d}, \mathrm{e}, \mathrm{f}=\mathrm{P}<0.001$ if compared with each other groups except and $(\mathrm{G} 3 \alpha \mathrm{G} 4 \mathrm{~A})=\mathrm{P}<0.05$. Groups with the same superscript letter mean no significant difference.

\section{Discussion}

Induction of xenograft MCF-7 estradiol causes hepatotoxicity in an animal model and the main cause of this toxicity is estradiol [39]. Toxicity study revealed a low level of or improvement of hepatic and nephrotoxicity of pip, BV and Hes the in case of single or in combination therapy with/without as compared with untreated breast cancer group which indicated by lower levels of AST, ALT, ALP, urea, and creatinine, especially in four 
combination treatment. Tam has a nonsignificant change in the level of liver enzymes as compared with untreated group. These results are agreed with the previous investigation as the following:

The investigation that to cast more light on the toxic effect of tamoxifen on some liver enzymes in mice representing metabolic pathways is glycolysis, gluconeogenesis and glycogenolysis and the role of vitamin $\mathrm{C}$ in minimizing the toxicity induced by tamoxifen. Results showed that a significant increase in alkaline phosphatase (ALP) in treated groups, while AST and ALT enzymes activities, urea, and creatinine showed a significant reduction in treated mice as compared to the normal healthy control group [48].

Another study showed that Tamoxifen induces hepatotoxicity and changes to hepatocyte morphology at the early stage of endocrine therapy in mice, and this is known to occur mainly due to the leakage of these enzymes from the damaged hepatocytes into the bloodstream [49].

Previous studies of piperine as the study investigated the effect of piperine on liver damage and bone changes caused by bile duct ligation in rats showed Piperine administered at 20,40 or $80 \mathrm{mg} / \mathrm{kg}$ to bile duct-ligated (BDL) rats reduced elevated plasma ALT, AST and ALP this may due to piperine a legend for the vanilloid receptor (TRPV1) reduced hepatic injury [50]. Also, another study was to investigate the antineoplastic activity of a combination of thymoquinone (TQ) and piperine against breast cancer implanted in mice and the results showed that normal serum levels of AST, ALT, and creatinine were observed in tumor-bearing mice treated with the combination therapy [51] This might be through its direct action on free radicals of lead acetate, protecting the kidney from cellular damage by maintaining its membrane integrity [52].

The effect BV on liver and kidney parameters investigated in the previous studies as that to evaluate the tumor growth inhibiting effects of bee venom in rats (in vivo) and in tumor cell cultures (in vitro), the level of AST, ALT, ALP significantly $(\mathrm{p} \leq 0.05)$ decreased in Ehrlich ascites carcinoma bearing rats treated groups hepato-protective effect of BV, Previous investigations of hesperidin were concomitant with this study as the following: Study was designed to explore the hepatoprotective and antioxidant effect of hesperidin, against gamma-irradiation which induced oxidative damage in the liver of rats. This may due to hesperidin tends to prevent liver damage, suppresses the leakage of enzymes through cellular membranes, preserves the integrity of the plasma membranes and hence restores these enzymes levels, Also ability to scavenge reactive oxygen species thereby preventing further peroxidation of membrane lipids, ability to enhance glutathione production by providing more substrate for reactive intermediates that promote detoxification mechanisms, restored the activities of the antioxidant enzymes, Thus, hesperidin contributes significantly to the intracellular antioxidant defense system by acting as a powerful consumer of superoxide anion and hydroxyl radicals [53]. a study designed to investigate the possible protective effects of multiple doses of hesperidin against cisplatin-induced nephrotoxicity induced by single i.p injection of cisplatin showed significantly reduced cisplatin-induced elevations in serum creatinine and BUN levels [54].In this study induction of breast cancer causes a significant increase in the MDA and decrease in SOD enzyme. The most commonly used assay for the evaluation of the intensity of lipid peroxidation is the analysis of the content of TBARS represented by MDA. Higher MDA content was also detected in breast cancer tissue when compared with healthy surrounding tissue, thus suggesting that ROS are produced in tumor tissues [40]. SOD activity measured in experimental animals in the present study may suggest that an excess in ROS production appeared in rats with mammary gland tumors. The increase of antioxidative enzyme activities is the organism's response to oxidative stress [41]. At present, it is difficult to determine whether the ROS increase was a primary factor in relation to the carcinogenic process, or it was the result of ROS generation in tumor cells. It is known that some cancer cells can produce ROS [40]. SOD catalyzes the dismutation of superoxide anion radical to oxygen and hydrogen peroxide [42], resulting in the increase of hydrogen peroxide. Thus, 
protection against ROS requires GSH-Px and catalase activities which neutralize hydrogen peroxide.

In the present study, there was a depletion of SOD and elevation of MDA in tumorous mammary gland as compared to the normal group. Administration of tam and other three natural products alter the concentration of SOD and MDA as compared to untreated group. Results of this study were agreed with previous research such as the following:

A study that detected the effects of the combination between tamoxifen and thymoquinone in patients with breast cancer. This study showed that Tam or thymoquinone alone or in combination induced a significant increase in tumor SOD with a significant decrease in the relapse rate, tumor tissue MDA. This effect may due to TAM has an antioxidant effect through increasing the activities of the antioxidant enzymes and decreasing lipid peroxidation products [43].

The study was to determine the protective effect of Piper aduncum capsules on DMBA (dimethylbenz $[\alpha]$ anthracene)-induced breast cancer in rats. Results showed significantly low level of MDA and elevated level of SOD implicates potent antioxidant effect.P. aduncum capsule contains saponins, polyphenols, tannins, alkaloids, and flavonoids in significant quantities. The effects of the capsule could be due to its ability to reduce oxidative stress and free-radical formation since it contains flavonoids. Also, flavonoids have effects on aromatase, a mitochondrial cytochrome P450 family enzyme produced at high levels in breast tissues that catalyzes the conversion of androgens to estrogens [44].

Previous investigations of hesperidin such as one that evaluated the therapeutic potential of hesperidin by assaying the activities of antioxidant enzymes, lipid peroxidation in anthracene- induced breast cancer. Daily oral administration of hesperidin $(30 \mathrm{mg} / \mathrm{kg}$ body $\mathrm{wt})$ to breast cancer-bearing rats. The result showed a significant decrease in the level of SOD and MDA [45]. Hesperidin significantly protects from DNA, lipids, and proteins against free radical damage. The antioxidant mechanism of hesperidin was planned by hydrogen donation to free radicals and the formation of a complex between the lipid radical and the antioxidant radical. The presences of 3-o-hydroxy, 4-o-methoxy system in the B ring are necessary for radical scavenging properties of flavonoids, which possess electron donating properties and are a radical target. Also, electron delocalization from the $\mathrm{B}$ ring occurred by the $\mathrm{C} 4-\mathrm{C} 8$ double bond conjugated with a 4-keto group. The presence of both $3-\mathrm{OH}$ and $5-\mathrm{OH}$ groups, in combination with a 4-carbonyl function and C40 -C80 double bond, increases the radical scavenging activity. Flavonols that include more hydroxyl groups (1 to $6 \mathrm{OH}$ groups) are potent free radical and superoxide anion radical scavengers, such as hesperidin. Hesperidin has a 2-hydroxyl group on the A and $\mathrm{B}$ rings and on methoxy group on the $\mathrm{B}$ ring, in addition to possessing the $4^{\prime}, 8^{\prime}$-double bond in conjugation with a 4-oxo function of the $\mathrm{C}$ ring, which is responsible for potent radical scavenging activities of hesperidin [46].

Previous investigations of hesperidin such as one that was designed to investigate the therapeutic effects of bee venom (BV) on high-fat diet (HFD)-induced nonalcoholic fatty liver (NAFL) in rats at different levels. The results of this study, which indicated that NAFL rats treated with BV showed significantly lower hepatic levels of MDA compared to untreated rats in a dose-dependent manner [47].

\section{Conclusion}

The obtained in vivo results on xenograft MCF7 induced breast cancer in rats establish the efficacy of new drugs combination that improve the oxidative stress and hepatonephroprotective which a mean to fight breast cancer with low toxicity.

\section{References}

[1] D. Forman, J. Ferlay, B. Stewart, C. Wild, The global and regional burden of cancer, World cancer report 2014 (2014) 16-53.

[2] S. Palit, S. Kar, G. Sharma, P.K. Das, Hesperetin induces apoptosis in breast carcinoma by triggering accumulation of ROS and activation of ASK1/JNK pathway, Journal of cellular physiology 230(8) (2015) 1729-1739.

[3] Y. Deng, S. Sriwiriyajan, A. Tedasen, P. Hiransai, P. Graidist, Anti-cancer effects of Piper nigrum via inducing multiple molecular signaling in vivo and in vitro, Journal of ethnopharmacology 188 (2016) 87-95. 
[4] M. Dowsett, J. Cuzick, J. Ingle, A. Coates, J. Forbes, J. Bliss, M. Buyse, M. Baum, A. Buzdar, M. Colleoni, Meta-analysis of breast cancer outcomes in adjuvant trials of aromatase inhibitors versus tamoxifen, Journal of Clinical Oncology 28(3) (2009) 509-518.

[5] F. Petrelli, A. Coinu, M. Cabiddu, M. Ghilardi, V. Lonati, S. Barni, Five or more years of adjuvant endocrine therapy in breast cancer: a metaanalysis of published randomised trials, Breast cancer research and treatment 140(2) (2013) 233 240.

[6] C. Davies, H. Pan, J. Godwin, R. Gray, R. Arriagada, V. Raina, M. Abraham, V.H.M. Alencar, A. Badran, X. Bonfill, Long-term effects of continuing adjuvant tamoxifen to 10 years versus stopping at 5 years after diagnosis of oestrogen receptor-positive breast cancer: ATLAS, a randomised trial, The Lancet 381(9869) (2013) 805-816.

[7] B.P. Kumar, S. Rajput, K.K. Dey, A. Parekh, S. Das, A. Mazumdar, M. Mandal, Celecoxib alleviates tamoxifen-instigated angiogenic effects by ROSdependent VEGF/VEGFR2 autocrine signaling, BMC cancer 13(1) (2013) 273.

[8] E.A. Musgrove, R.L. Sutherland, Biological determinants of endocrine resistance in breast cancer, Nature Reviews Cancer 9(9) (2009) 631643.

[9] J.D. Lewis, A.B. Chagpar, E.A. Shaughnessy, J. Nurko, K. McMasters, M.J. Edwards, Excellent outcomes with adjuvant toremifene or tamoxifen in early stage breast cancer, Cancer 116(10) (2010) 2307-2315.

[10] E.-S.M. El-Sayed, O.M. Abo-Salem, M.F. AbdEllah, G.M. Abd-Alla, Hesperidin, an antioxidant flavonoid, prevents acrylonitrile-induced oxidative stress in rat brain, Journal of biochemical and molecular toxicology 22(4) (2008) 268-273.

[11] C.J. Lee, L. Wilson, M.A. Jordan, V. Nguyen, J. Tang, G. Smiyun, Hesperidin suppressed proliferations of both Human breast cancer and androgen-dependent prostate cancer cells, Phytotherapy research 24(S1) (2010).

[12] T. Tanaka, H. Makita, K. Kawabata, H. Mori, M. Kakumoto, K. Satoh, A. Hara, T. Sumida, T. Tanaka, H. Ogawa, Chemoprevention of azoxymethane-induced rat colon carcinogenesis by the naturally occurring flavonoids, diosmin and hesperidin, Carcinogenesis 18(5) (1997) 957-965.

[13] M. Yang, T. Tanaka, Y. Hirose, T. Deguchi, H. Mori, Y. Kawada, Chemopreventive effects of diosmin and hesperidin on $\mathrm{N}$-butyl- N-(4hydroxybutyl) nitrosamine-induced urinarybladder carcinogenesis in male ICR mice, International journal of cancer 73(5) (1997) 719724.

[14] I.-Y. Choi, S.-J. Kim, H.-J. Jeong, S.-H. Park, Y.-S. Song, J.-H. Lee, T.-H. Kang, J.-H. Park, G.-S. Hwang, E.-J. Lee, Hesperidin inhibits expression of hypoxia inducible factor-1 alpha and inflammatory cytokine production from mast cells, Molecular and cellular biochemistry 305(1-2) (2007) 153-161.
[15] H. Park, M.-J. Kim, E. Ha, J.-H. Chung, Apoptotic effect of hesperidin through caspase 3 activation in human colon cancer cells, SNU-C4, Phytomedicine 15(1) (2008) 147-151.

[16] M.-H. Yeh, S.-T. Kao, C.-M. Hung, C.-J. Liu, K.-H. Lee, C.-C. Yeh, Hesperidin inhibited acetaldehydeinduced matrix metalloproteinase-9 gene expression in human hepatocellular carcinoma cells, Toxicology letters 184(3) (2009) 204-210.

[17] A. Samykutty, A.V. Shetty, G. Dakshinamoorthy, M.M. Bartik, G.L. Johnson, B. Webb, G. Zheng, A. Chen, R. Kalyanasundaram, G. Munirathinam, Piperine, a bioactive component of pepper spice exerts therapeutic effects on androgen dependent and androgen independent prostate cancer cells, PloS one 8(6) (2013) e65889.

[18] K. Srinivasan, Black pepper and its pungent principle-piperine: a review of diverse physiological effects, Critical reviews in food science and nutrition 47(8) (2007) 735-748.

[19] C. Pradeep, G. Kuttan, Piperine is a potent inhibitor of nuclear factor $-\kappa B(N F-\kappa B), c-F o s$, CREB, ATF-2 and proinflammatory cytokine gene expression in B16F-10 melanoma cells, International immunopharmacology 4(14) (2004) 1795-1803.

[20] E. Sunila, G. Kuttan, Immunomodulatory and antitumor activity of Piper longum Linn. and piperine, Journal of ethnopharmacology 90(2) (2004) 339-346.

[21] C. Pradeep, G. Kuttan, Effect of piperine on the inhibition of lung metastasis induced B16F-10 melanoma cells in mice, Clinical \& experimental metastasis 19(8) (2002) 703-708.

[22] J.-E. Huh, Y.-H. Baek, M.-H. Lee, D.-Y. Choi, D.S. Park, J.-D. Lee, Bee venom inhibits tumor angiogenesis and metastasis by inhibiting tyrosine phosphorylation of VEGFR-2 in LLC-tumorbearing mice, Cancer letters 292(1) (2010) 98110.

[23] M.-H. Jang, M.-C. Shin, S. Lim, S.-M. Han, H.-J. Park, I. Shin, J.-S. Lee, K.-A. Kim, E.-H. Kim, C.J. Kim, Bee venom induces apoptosis and inhibits expression of cyclooxygenase-2 mRNA in human lung cancer cell line NCI-H1299, Journal of pharmacological sciences 91(2) (2003) 95-104.

[24] S.-J. Hong, G.S. Rim, H.I. Yang, C.S. Yin, H.G. Koh, M.-H. Jang, C.-J. Kim, B.-K. Choe, J.-H. Chung, Bee venom induces apoptosis through caspase-3 activation in synovial fibroblasts of patients with rheumatoid arthritis, Toxicon 46(1) (2005) 39-45.

[25] C. Wang, T. Chen, N. Zhang, M. Yang, B. Li, X. Lü, X. Cao, C. Ling, Melittin, a major component of bee venom, sensitizes human hepatocellular carcinoma cells to tumor necrosis factor-related apoptosis-inducing ligand (TRAIL)-induced apoptosis by activating CaMKII-TAK1-JNK/p38

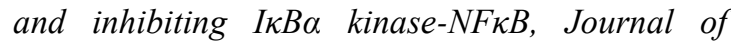
Biological Chemistry 284(6) (2009) 3804-3813.

[26] D.-O. Moon, S.-Y. Park, M.-S. Heo, K.-C. Kim, C. Park, W.S. Ko, Y.H. Choi, G.-Y. Kim, Key regulators in bee venom-induced apoptosis are Bcl-2 and caspase-3 in human leukemic U937 cells through downregulation of ERK and Akt, 
International immunopharmacology 6(12) (2006) 1796-1807.

[27] S.-W. Ip, S.-S. Liao, S.-Y. Lin, J.-P. Lin, J.-S. Yang, M.-L. Lin, G.-W. Chen, H.-F. Lu, M.-W. Lin, S.-M. Han, The role of mitochondria in bee venominduced apoptosis in human breast cancer MCF7 cells, In Vivo 22(2) (2008) 237-245.

[28] M.H. Park, M.S. Choi, D.H. Kwak, K.W. Oh, D.Y. Yoon, S.B. Han, H.S. Song, M.J. Song, J.T. Hong, Anti-cancer effect of bee venom in prostate cancer cells through activation of caspase pathway via inactivation of $N F-\kappa B$, The Prostate 71(8) (2011) 801-812.

[29] M. Jo, M.H. Park, P.S. Kollipara, B.J. An, H.S. Song, S.B. Han, J.H. Kim, M.J. Song, J.T. Hong, Anti-cancer effect of bee venom toxin and melittin in ovarian cancer cells through induction of death receptors and inhibition of JAK2/STAT3 pathway, Toxicology and applied pharmacology 258(1) (2012) 72-81.

[30] NRC, Guide for the care and use of laboratory animals. Institute of Laboratory Animal Resources, Commission on Life Sciences, National Academy of Sciences, Washington, DC (1996).

[31] R. Dunpall, A. Opoku, N. Revaprasadu, Development and characterization of MCF7 mammary carcinoma xenografts in a nonimmunocompromised rat model, Tropical Journal of Pharmaceutical Research 15(10) (2016) 2085. 2091.

[32] R.V. Ginckel, B. Janssens, M. Callens, N. Goeminne, L. Wouters, R.D. Coster, Effects of combined and sequential treatment with tamoxifen and the aromatase inhibitor vorozole on 7, 12dimethylbenz (a) anthracene-induced mammary carcinoma in the rat, Cancer Chemotherapy and Pharmacology 38(1) (1996) 21-28.

[33] S. Belhan, M. ÖZKARACA, F.M. Kandemir, Effectiveness of Hesperidin on MethotrexateInduced Testicular Toxicity in Rats, biological membranes 4 (2017) 6.

[34] P. Sankar, A. Telang, A. Manimaran, Effect of piperine on cypermethrin-induced oxidative damage in rats, J Vet Sci Technol 2(1) (2011) 1-5.

[35] J. Choi, C. Jeon, J.H. Lee, J.U. Jang, F.S. Quan, K. Lee, W. Kim, S.K. Kim, Suppressive Effects of Bee Venom Acupuncture on Paclitaxel-Induced Neuropathic Pain in Rats: Mediation by Spinal $\alpha 2-$ Adrenergic Receptor, Toxins 9(11) (2017) 351.

[36] L. Karimzadeh, M. Nabiuni, A. Sheikholeslami, S. Irian, Bee venom treatment reduced $C$-reactive protein and improved follicle quality in a rat model of estradiol valerate-induced polycystic ovarian syndrome, Journal of Venomous Animals and Toxins including Tropical Diseases 18(4) (2012) 384-392.

[37] P. Kakkar, B. Das, P. Viswanathan, A modified spectrophotometric assay of superoxide dismutase, (1984).

[38] L. Mesbah, B. Soraya, S. Narimane, P. Jean, protective effect of flavonides against the toxicity of vinblastine cyclophosphamide and paracetamol by inhibition of lipid-peroxydation and increase of liver glutathione, Haematol 7(1) (2004) 59-67.
[39] G. Pandey, S. Pandey, M. Sharma, Experimental hepatotoxicity produced by ethinyl estradiol, Toxicology international 18(2) (2011) 160.

[40] O. Portakal, Ö. Özkaya, B. Bozan, M. Koşan, I. Sayek, Coenzyme Q10 concentrations and antioxidant status in tissues of breast cancer patients, Clinical biochemistry 33(4) (2000) 279284.

[41] M. Jurgowiak, K. Bialkowski, R. Olinski, Reaktywne formy tlenu a regulacja ekspresji genów, Postęy Biochemii 42(1) (1996) 6-13.

[42] D. Klug, J. Rabani, I. Fridovich, A direct demonstration of the catalytic action of superoxide dismutase through the use of pulse radiolysis, Journal of Biological Chemistry 247(15) (1972) 4839-4842.

[43] A. Kabel, M. El-Rashidy, M. Omar, Ameliorative Potential of Tamoxifen/Thymoquinone Combination in Patients with Breast Cancer: A Biochemical and Immunohistochemical Study, Cancer Med Anticancer Drug 1(102) (2016) 2.

[44] J. Arroyo-Acevedo, R. Chávez-Asmat, A. AnampaGuzmán, R. Donaires, J. Ráez-Gonzáles, Protective effect of Piper aduncum capsule on DMBA-induced breast cancer in rats, Breast cancer: basic and clinical research 9 (2015) BCBCR. S24420.

[45] N. Nandakumar, M.P. Balasubramanian, Hesperidin protects renal and hepatic tissues against free radical-mediated oxidative stress during DMBA-induced experimental breast cancer, Journal of Environmental Pathology, Toxicology and Oncology 30(4) (2011).

[46] A. Ahmadi, A. Shadboorestan, Oxidative stress and cancer; the role of hesperidin, a citrus natural bioflavonoid, as a cancer chemoprotective agent, Nutrition and cancer 68(1) (2016) 29-39.

[47] M.Y. Hanafi, E.L. Zaher, S.E. El-Adely, A. Sakr, A.H. Ghobashi, M.H. Hemly, A.H. Kazem, M.A. Kamel, The therapeutic effects of bee venom on some metabolic and antioxidant parameters associated with HFD-induced non-alcoholic fatty liver in rats, Experimental and Therapeutic Medicine 15(6) (2018) 5091-5099.

[48] Z. Zuhair, The role of vitamin $C$ in alteration of enzymes responsible of energy metabolism induced by administration of tamoxifen to mouse, Advances in Biological Chemistry 1(02) (2011) 15.

[49] F.F. Gao, J.W. Lv, Y. Wang, R. Fan, Q. Li, Z. Zhang, L. Wei, Tamoxifen induces hepatotoxicity and changes to hepatocyte morphology at the early stage of endocrinotherapy in mice, Biomedical reports 4(1) (2016) 102-106.

[50] O. Abdel Salam, Nofal, SM, El-Shenawy, SM, Shaffie, NM., Effect Of Piperine On Liver Damage And Bone Changes Caused By Bile Duct Ligation In Rats, The Internet Journal of Pharmacology 5 (2007) 2 .

[51] W.H. Talib, Regressions of Breast Carcinoma Syngraft Following Treatment with Piperine in Combination with Thymoquinone, Scientia pharmaceutica 85(3) (2017) 27.

[52] S.A. Sudjarwo, K. Eraiko, K. Giftania Wardani Sudjarwo, Protective effects of piperine on lead acetate induced-nephrotoxicity in rats, Iranian 
journal of basic medical sciences 20(11) (2017) 1227.

[53] K. Pradeep, S.H. Park, K.C. Ko, Hesperidin a flavanoglycone protects against $\gamma$-irradiation induced hepatocellular damage and oxidative stress in Sprague-Dawley rats, European journal of pharmacology 587(1) (2008) 273-280.

[54] W.R. Mohamed, E.-S.A. Arafa, B.A. Shehata, G.A. El Sherbiny, A.N.A. Elgendy, Beneficial Effects of Hesperidin against Cisplatin-Induced Nephrotoxicity and Oxidative Stress in Rats, British Journal of Pharmacology and Toxicology 6(3) (2015) 56-63. 